OPEN ACCESS

Edited by:

Christoph Scheiermann, Ludwig Maximilian University of

Munich, Germany

Reviewed by:

David Ray,

University of Oxford, United Kingdom

Anne Dudeck,

Universitätsklinikum

Magdeburg, Germany

${ }^{*}$ Correspondence:

Atsuhito Nakao

anakao@yamanashi.ac.jp

Specialty section:

This article was submitted to

Molecular Innate Immunity,

a section of the journal

Frontiers in Immunology

Received: 25 March 2020

Accepted: 18 May 2020

Published: 12 June 2020

Citation:

Nakao A (2020) Circadian Regulation of the Biology of Allergic Disease:

Clock Disruption Can Promote Allergy.

Front. Immunol. 11:1237.

doi: 10.3389/fimmu.2020.01237

\section{Circadian Regulation of the Biology of Allergic Disease: Clock Disruption Can Promote Allergy}

\author{
Atsuhito Nakao ${ }^{1,2 *}$ \\ ${ }^{1}$ Department of Immunology, Faculty of Medicine, University of Yamanashi, Kofu, Japan, ${ }^{2}$ Atopy Research Center, Juntendo \\ University School of Medicine, Tokyo, Japan
}

Allergic diseases such as allergic rhinitis, asthma, atopic dermatitis, and food allergy are characterized by epithelial barrier dysfunction and deregulated immune responses. Components of the circadian clock interact with critical elements of epithelial barrier function and immune responses, and regulate the biological processes on a 24-h cycle at steady state. This may represent an anticipatory defense response to day-night fluctuation of attack by noxious stimuli such as pathogens in the environment. This review will summarize clock control of epithelial barrier function and immune responses associated with allergic disease and offer novel insights and opportunities into how clock dysfunction impacts allergic disease. Importantly, perturbation of normal clock activity by genetic and environmental disturbances, such as chronic light cycle perturbations or irregular eating habits, deregulates epithelial barrier function and immune responses. This implies that the circadian clock is strongly linked to the fundamental biology of allergic disease, and that clock disruption can precipitate allergic disease by altering the epithelial barrier and immune functions. Given that contemporary lifestyles often involve chronic circadian disruptions such as shift work, we propose that lifestyle or therapeutic interventions that align the endogenous circadian clock with environmental cycles should be a part of the efforts to prevent or treat allergic disease in modern society.

Keywords: circadian clock, allergic disease, epithelial barrier function, immune response, clock disruption

\section{INTRODUCTION}

Allergic diseases such as allergic rhinitis, asthma, atopic dermatitis, and food allergy are serious public and medical concerns due to their high prevalence and the harm they cause, in terms of both patient quality of life and socioeconomics (1). However, we do not fully understand why allergic disease is so prevalent in modern society. Hence, we need to understand previously unrecognized aspects of the biology of allergic disease.

Allergic disease is characterized by epithelial barrier dysfunction and deregulated immune response (allergic immune response) (2-4). For instance, in atopic dermatitis (AD), several components of skin barrier function, such as filaggrin, tight junction, and the microbiome are compromised in terms of quantity and/or quality, leading to increased cutaneous permeability (5). Consequently, the skin permits allergen penetration and releases epithelial cytokines (e.g., IL-33), which trigger an allergic immune response. Decreased regulatory T-cell activity in AD may also promote allergic immune responses (6). 
The circadian clock is the endogenous timing-keeping mechanisms, by which living organisms fit their physiology and behavior to daily alterations in the rhythmic environment created by the earth's rotation (7). Recent studies highlight that the circadian clock underpins epithelial barrier function and immune responses under physiological conditions (8-12). The components of the circadian clock interact with critical elements or pathways of epithelial barrier function and immune responses, thereby regulating these biological processes on a 24$\mathrm{h}$ cycle. This may represent an anticipatory defense response to day-night fluctuation of attack by pathogens and nonpathogenic insults in the outer environment $(11,13)$. In this review, I will summarize clock control of epithelial barrier function and immune responses that are associated with allergic disease and provide insight into how clock dysfunction affects allergic disease. Allergic disease is also well-characterized by marked day-night changes in the clinical symptoms, laboratory parameters, and response to treatment. Readers who are interested in this subject are encouraged to refer to the reviews on how the circadian clock underpins a time of day-dependent variation in allergic reactions (14-16). The reviews highlight that oscillatory allergic reactions are generated by rhythmic expression of key molecules in the pathophysiology controlled by the circadian clock.

\section{MOLECULAR CLOCKS IN MAMMALIAN CELLS}

In mammals, the circadian clock consists of several clock genes that are expressed in virtually all cell types, including cells in the skin, gut, airways, and immune system $(11,12,17-$ 19). At its core, the molecular clock consists of interlocking transcriptional-translational feedback loops (TTFLs) centered on the transcription factors BMAL1 and CLOCK $(20,21)$. BMAL1 heterodimerizes with CLOCK, and the heterodimer binds to E-box motifs (CANNTG) throughout the genome, driving the expression of thousands of genes, including Period (Per1-3) and Cryptochrome (Cry1,2). The PER and CRY proteins form oligomers and move to the nucleus, where they inhibit BMAL1/CLOCK activity. This core loop, which takes $\sim 24 \mathrm{~h}$ to complete, involves several post-transcriptional mechanisms such as enzymatic degradation of PER and CRY, and acts as a molecular oscillator to represent time of day within each cell.

Other than this core loop, a stabilizing loop within the clockwork regulating the timing and amplitude of BMAL1 is provided by the nuclear receptors $\operatorname{ROR} \alpha$ and REV-ERB $\alpha$ (Nr1d1) (20, 21). The BMAL1/CLOCK heterodimer activates transcription of $\operatorname{ROR} \alpha$ and $\operatorname{REV}-\mathrm{ERB} \alpha$, which respectively activates and represses BMAL1 transcription.

Accordingly, the network of circadian proteins mediates periodic expression of thousands of genes (clock-controlled genes: CCGs) and regulates the timing of cellular activities on a 24-h cycle, ultimately dictating rhythmic physiology in various organs. The impact of the clockwork machinery is enormous: many CCGs, such as nuclear hormone receptors
(NRs) like glucocorticoid receptor, are key regulators of major physiological processes (e.g., metabolism, immunity, development, reproduction) (22). Overall, 43\% of all protein coding genes in mice shows circadian rhythms in transcription somewhere in the body, in an organ-specific manner (23).

\section{COORDINATION OF THE MULTI-OSCILLATORS}

The human body contains $\sim 40$ trillion cells, each with a $\sim 24$-h clock. Thus, our body consists of a multi-oscillator network system. How are these astronomical numbers of clocks synchronized with other?

In mammals, the suprachiasmatic nucleus (SCN) of the hypothalamus (the central clock) serves as the master pacemaker in the body $(20,21,24)$. The SCN receives afferent innervation about environmental light levels from the retina via the retinohypothalamic tract, which synchronizes the SCN clock via the cAMP response element binding (CREB) protein. In turn, the SCN transmits neuronal (e.g., sympathetic nerve activity) and hormonal (e.g., cortisol) signaling to synchronize peripheral clocks.

However, peripheral clocks can also be reset by non-SCNderived hormones (e.g., insulin) and metabolic (e.g., $\mathrm{NAD}^{+}$ [nicotinamide adenine dinucleotide] ${ }^{+}$) signals connected with non-photic environmental cues such as food timing (25). For instance, livers of mice fed exclusively during the night or ad libitum (active phase) show a similar phase angle of cyclic liver gene expression, whereas feeding during the day almost entirely inverts the phase of liver oscillatory gene expression (26).

\section{HOW DOES CIRCADIAN DISRUPTION OCCUR?}

As stated, circadian clocks become synchronized to a 24-h periodic environmental cue, which is called the zeitgeber ("timegiver" in German). Light and meal timing are strong zeitgebers. Accordingly, circadian rhythms in behavior, physiology, and metabolism become robust when the rhythmicity of internal clocks is coupled to that of external zeitgebers (20, 21, $24,25)$. In other words, rhythms in the circadian system dampen when internal clock timing becomes mismatched with environmental zeitgebers.

Misalignment between the endogenous circadian clock and environmental cycles (or zeitgebers) compromises human mental and physical health (27). For instance, chronic circadian misalignment via night shift work, jet lag, or exposure to artificial light at night can precipitate or exacerbate mood disorders in susceptible individuals (28). Notably, night shift workers, who are exposed to aberrant light/dark conditions, irregular eating habits, and sleep disruption, are at a higher risk of cancer, cardiovascular and metabolic diseases, as well as sleep/psychiatric disorders (29). Thus, dramatic changes in modern lifestyles, including night shift work, nocturnal feeding, and shortness or irregularity of sleep impair our health via chronic circadian misalignment. 


\section{CIRCADIAN REGULATION OF THE BIOLOGY OF ALLERGIC DISEASE \\ Clock Control of Epithelial Barrier Function Related to Allergic Disease}

The epithelium in the skin, intestine, and airways acts as a physical, chemical, and biological barrier against pathogens, chemical agents, and allergens. Epithelial barrier dysfunction is critical for the initiation of allergic disease in many organs $(2,3,5)$. In brief, disruption of epithelial barrier function increases epithelial permeability that enables entry of allergens into the body and activates the allergic immune response. Genetic (e.g., filaggrin deficiency) and non-genetic factors (e.g., protease activity of allergens, chemical agents, and injury/itch) contribute to the barrier disruption associated with allergic disease (30).

Below, I briefly summarize some examples of clock control of epithelial barrier function related to allergic disease. I will not discuss clock control of commensal bacteria, an important barrier against pathogenic and non-pathogenic insults in the epithelium of the skin, intestine, and airways, because this subject has been adequately reviewed elsewhere (31-33).

\section{Skin}

Our largest organ, the skin, is continuously exposed to numerous environmental irritants, including allergens. The skin barrier consists of many physical, chemical, and biological components, including filaggrin, lipid (e.g., ceramide), skin $\mathrm{pH}$, tight junctions (TJs), anti-microbial peptides, commensal bacteria, and water content, most of which exhibit circadian rhythms $(3,34)$. In atopic dermatitis (AD), skin barrier function is impaired by several mechanisms, including filaggrin deficiency, injury (itch), and type 2 cytokines (e.g., IL-4 and IL-13) altering TJ protein expression, thereby precipitating allergic sensitization and inflammation $(34,35)$.

A clear example of circadian control of skin barrier function is aquaporin-3 (AQP3), which regulates water content by facilitating water and glycerol entry into keratinocytes. AQP3 expression in the skin is temporally controlled by CLOCK in mice and humans (36). Accordingly, stratum corneum hydration exhibits a significant $24-\mathrm{h}$ rhythm in wild-type mice that is absent in Clock-mutated mice. Interestingly, Clock-mutated mice exhibit persistently reduced stratum corneum hydration (36). These findings illustrate that clock disruption can deregulate the components of skin barrier function.

\section{Intestine}

Intestinal epithelial cells function as the first line of defense against pathogenic and non-pathogenic microorganisms in the gut lumen (37). These epithelial barriers include TJs, mucus, and anti-microbial peptides. Several reports in humans and mice suggest that food allergy is associated with intestinal barrier dysfunction, which increases intestinal permeability to food compounds (38).

Several studies report that intestinal barrier function is under the circadian control. The epithelial TJ is a multiprotein complex that forms a selectively permeable seal between adjacent epithelial cells and limits paracellular passage of macromolecules, including allergens (39). CLOCK regulates expression of the TJ molecules Occludin and Claudin-1 in the mouse colon and controls circadian periodicity of intestinal permeability (40). In addition, in a mouse model of ovalbumin (OVA)-induced food allergy, OVA-induced allergic diarrhea exhibits daily variations associated with circadian periodicity in intestinal permeability (41), which implicates that the timing of food antigen intake can affect the severity of food allergy symptoms via clock control of intestinal permeability.

Importantly, Clock-mutated mice express lower levels of Occludin and Claudin-1 in the colon and are more sensitive than wild-type mice to the colonic injury induced by dextran sodium sulfate (DSS) (40). In addition, in a mouse model of chronic alcohol feeding, circadian disruption through genetics (Clock mutation) or environmental disruption (weekly 12-h phaseshifting) results in gut leakiness and exacerbates alcohol-induced gut leakiness and liver pathology (42). These findings illustrate that clock disruption can impair intestinal barrier integrity.

\section{Airways}

The airways are continuously exposed to physical, chemical, and biological insults in the air, including pathogens, pollutants, and allergens, and protect the host against them. Like the skin and intestine, the airway epithelial barrier consists of many physical, chemical, and biological components, including TJs, mucus-producing goblet cells, ciliated cells, and epithelialderived cytokines/chemokines. Defects in the epithelial barrier are associated with asthma: for instance, bronchial biopsies from patients with asthma exhibit patchy disruption of TJs (43). Furthermore, these patients are more susceptible than healthy people to cigarette smoke or viral infection, which may precipitate asthma (44).

Pulmonary epithelial cells release cytokines and chemokines upon exposure to bacteria and viruses as a part of an innate defense response. The LPS-induced lung inflammatory response exhibits circadian periodicity in mice, relying on Bmall in bronchial epithelial cells (45). Since the epithelial response to LPS in the lung determines the development and severity of asthma $(46,47)$, Bmal1 regulation of the epithelial response to LPS may potentially explain the circadian nature of this disease.

Importantly, Bmal1 deletion in bronchial epithelial cells enhances the LPS response in the lung without time-of-day oscillations (45). In addition, a loss-of-function mutation of REV$\mathrm{ERB} \alpha$, a downstream target of Bmal1, in bronchial epithelial cells augments the LPS-induced inflammation in the lung, suggesting REV-ERB $\alpha$ as a key molecule that couples the core clock gene Bmall to innate immunity in the lung (48). Furthermore, responses to influenza infection are disrupted in mice with selective Bmall deletion in bronchial epithelial cells (49). Thus, clock disruption can deregulate airway defense (barrier) mechanisms.

\section{Clock Control of Innate Immune Responses Related to Allergic Disease Mast Cells/Basophil Response}

Mast cells and basophils are innate immune cells that share many features, although they constitute a distinct lineage 
having several different roles in immune response and tissue homeostasis. In IgE-mediated allergic diseases such as allergic rhinitis, asthma, and food allergy, mast cells and basophils are the main effector cells and are activated by an IgE-dependent mechanism. IgE produced in response to allergen binds to the high-affinity IgE receptor (FceRI) on the cell surface of mast cells and basophils, which triggers degranulation and production of cytokines/chemokines and lipid mediators, thereby shaping allergic inflammation (50).

The circadian nature of IgE-mediated allergic diseases is welldocumented: in allergic rhinitis and asthma, symptoms, nasal or bronchial reactivity, and inflammatory activity become more pronounced in the early morning and at midnight (51). Skin hypersensitivity to allergens also varies with the time of day (52). These findings suggest that IgE-mediated allergic disease is under circadian control.

Consistent with the clinical observations, CLOCK controls the expression of FceRI in mouse mast cells and releases mediators in a circadian manner upon IgE stimulation (53). Human basophils isolated from patients with asthma or allergic rhinitis exhibit a time-of-day-dependent variation in IgE-mediated responses (54, 55), although these findings may be controversial (56). In vivo, the extent of the passive cutaneous anaphylactic (PCA) reaction, a classical rodent model of IgE/mast cell-mediated allergic reaction, exhibits circadian variations $(53,57,58)$.

Interestingly, the extent of the PCA reaction in Per2-mutated mice, mast cell-selective Clock-mutated mice, or mice fed only in the resting phase persistently exhibits a peak level equivalent to that of wild-type mice, but without circadian periodicity (53, 57-59). Thus, circadian disruption due to genetic alteration or irregular eating habits increases the susceptibility of mast cells to IgE throughout the day.

IL-33 activates mast cells and basophils via its receptor ST2. The crucial roles of the IL-33/ST2 axis in both IgE- and non-IgEmediated allergic disease have been appreciated (60). CLOCK temporally gates the mast cell and basophil response to IL33 via regulation of ST2 expression, which may also underlie circadian nature of allergic disease (61). Importantly, Clock mutation persistently enhances their response to IL-33 (61). Thus, clock disruption may enhance IgE- or IL-33-mediated mast cell/basophil responses.

\section{Eosinophil Response}

Eosinophils are main effector cells for the control of parasitic infections, but increasing evidence suggests that they play regulatory roles in tissue homeostasis/repairs and adaptive immune response. In allergic disease, eosinophils mediate their effector functions through several mechanisms: degranulation, extracellular traps, and cytolysis (62). Eosinophils require IL5 survival signals, and anti-eosinophil monoclonal antibodies targeting IL-5 or IL5 receptor (IL5R) have been approved for clinical use against eosinophilic asthma (63).

Human eosinophils exhibit circadian oscillations in basal ECP (eosinophil cationic protein) expression and IL-8 (CXCL8) and CCL2 (The chemokine [C-C motif] ligand 2) release upon fMLP (N-formyl-methionyl-leucyl-phenylalanine) stimulation $(64,65)$. Furthermore, the number of blood and sputum eosinophils or serum IL-5 exhibits a time-of-day-dependent variation in asthma and control subjects or in mice (66-68). Interestingly, circadian variation of blood eosinophils has been linked to neuroendocrine and metabolic cycling (68). These findings are consistent with circadian control of eosinophil homeostasis, activation, and migration under steady states and in allergic disease, although few studies have addressed the direct roles of the clock in eosinophil function.

\section{Macrophage Response}

Macrophages are versatile innate immune cells that have phenotypic diversity and function in many different aspects of physiology and disease. Generally, the roles of macrophages in allergic disease remain obscure, but, in allergic asthma, macrophages may promote inflammatory responses associated with lung injury, fibrosis, and goblet cells hyperplasia (69).

Circadian clock components regulate various functions in macrophages, including cytokine secretion upon LPS challenge (70-72). About $8 \%$ of genes expressed in peritoneal macrophages are rhythmically transcribed, including essential elements in LPS/TLR4 signaling (71).

Interestingly, mice with Bmall-deficient myeloid cells have markedly elevated eosinophil accumulation and serum BALF IL-5 expression in a model of allergic asthma (73). Bmal1-deficient macrophages produce more asthma-associated CCL2 and CXCL10 upon LPS stimulation than wild-type macrophages. Further, targeting REV-ERB $\alpha$ in myeloid cells exhibits persistently high pulmonary neutrophilic inflammation upon LPS challenge without time-of-day-dependent variations (48). Thus, clock disruption may enhance asthma by altering responses to LPS in macrophages, as well as in bronchial epithelial cells.

\section{Innate Lymphoid Cell Response}

Innate lymphoid cells (ILCs) are the most recently discovered family of innate immune cells that are ubiquitously distributed and are enriched in mucosal surface. ILCs consist of three different groups: group1, group 2, and group 3 ILCs (ILC1s, ILC2s, ILC3s), based on transcription factors required for the development, cytokine expression, and distinct effector functions.

ILC2s are key regulators of allergic immune responses (74). The epithelium-derived cytokines IL-25, IL-33, and TSLP activate ILC2s. The activated ILC2s release IL-5 and IL-13, which initiate and amplify allergic inflammation by activating eosinophils and epithelial cells. ILC2s in blood are more abundant in patients with asthma than in healthy subjects (75). The roles of clock in ILC2s remain unclear, but it is highly likely that ILC2s development and function are under circadian control, since $\operatorname{ROR} \alpha$, a component of circadian clock, is involved in ILC2 differentiation (76) and IL-5 production from intestinal ILC2s exhibits circadian rhythms associated with blood eosinophil counts (68).

In contrast to ILC2s, several studies suggest that ILC3s, which provide mucosal defense through IL-22 and IL-17, are under strong control of the circadian clock. Bmall deficiency in ILC3s or disruption of light-dark cycles and feeding rhythms can deregulate gut ILC3 homeostasis, impairs epithelial reactivity, 


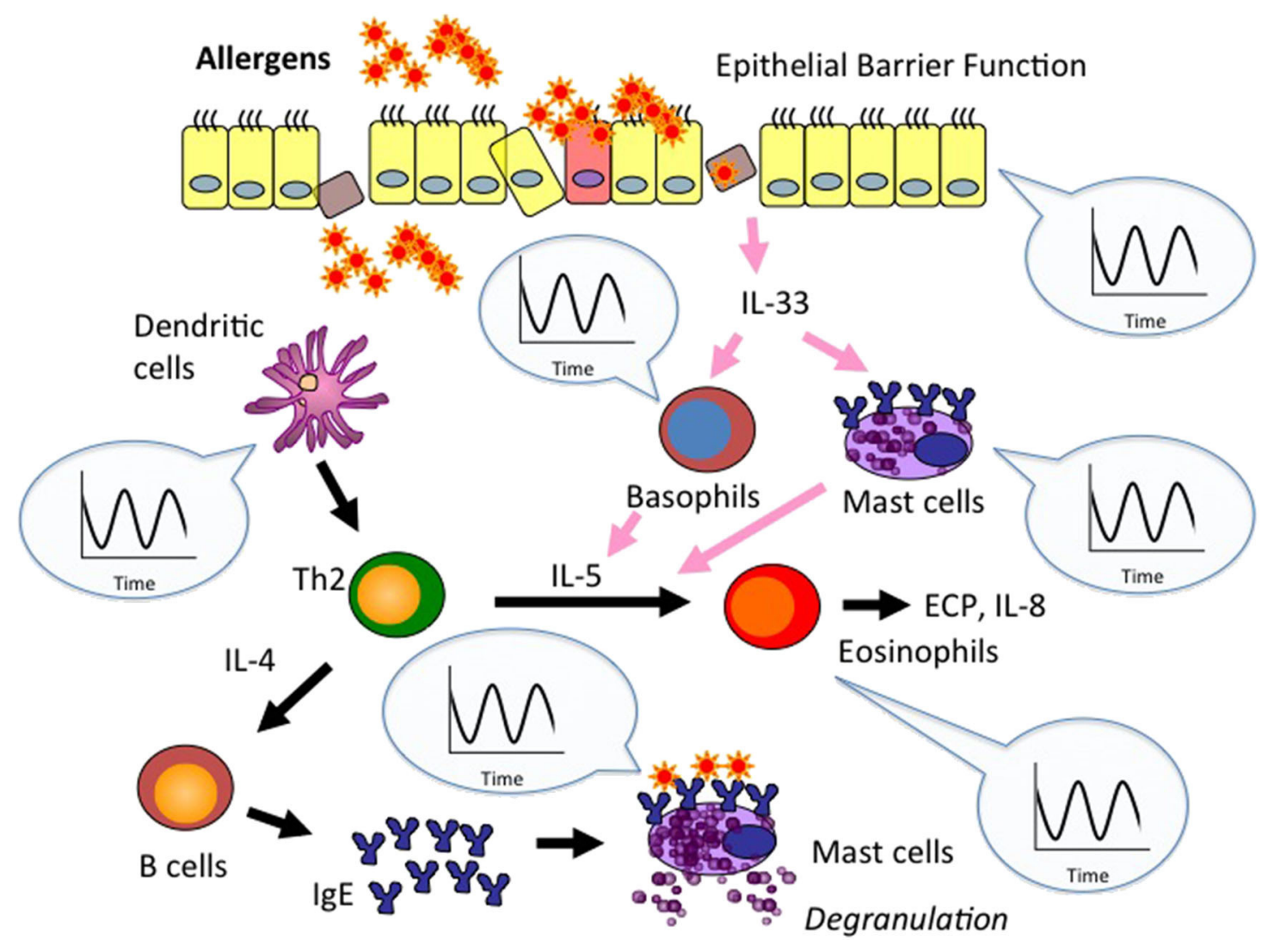

FIGURE 1 | Circadian clock activity is embedded in epithelial barrier function and immune response associated with allergic disease. Epithelial barrier disruption enables entry of allergens into the body and leads to production of innate cytokines such as IL-33. The cytokines skew dendritic cell (DC) phenotypes, activate innate immune cells (e.g., mast cells, basophils), and promote Th2 development. Th2 cells secrete IL-4 and IL-5, which promote IgE production from B cells and eosinophil accumulation, respectively, and shape allergic inflammation. As stated in the text, circadian clock activity is embedded in the control of epithelial barrier function and immune cell responses associated with allergic disease. Circadian control of allergy-related epithelial barrier functions and innate immune cell responses (e.g., mast cells, basophils, eosinopihls, dendritic cells) has been extensively studied so far. Thus, we emphasize clock control of these elements in this figure. Please note that macrophage-, innate lymphoid cell-, T cell-, and B cell-responses are also controlled by the circadian clock. Accordingly, clock disruption may predispose allergic disease by deregulating epithelial barrier function and immune cell responses.

and deregulates the microbiome $(77,78)$. Because ILC3s have been implicated in obesity-induced asthma (79), clock disruption through metabolic disturbances in obese patients might enhance ILC3 responses and predispose patients to asthma.

\section{Dendritic Cell Response}

Dendritic cells (DCs) are the most potent professional antigenpresenting cells (APCs) in vivo, which can induce the activation and differentiation of naive $\mathrm{T}$ cells or induce immune tolerance. The ability of DCs to trigger immunity or tolerance by their co-stimulatory/inhibitory molecules is likely involved in the development of allergic disease. For instance, DC-expressing semaphorin 4, one member of the large family of secreted and membrane-bound glycoproteins that were initially implicated in axon guidance and neural development, drives Th2 response and are highly expressed in human asthmatic lung tissue (80).

The roles of clock in DCs remain largely unclear. One study shows that deletion of Bmal1 in dendritic cells enhances the gut helminth Trichuris muris egg-specific protective Th2 response while suppressing the Th1 response in mice, suggesting that circadian machinery in DCs may contribute to Th1/Th2 balance (81). Thus, disruption of the DC-clock may affect Th1/Th2 balance, thereby having impact on allergic disease.

\section{Clock Control of Adaptive Immune Responses Related to Allergic Diseases}

Lymphocytes ( $\mathrm{T}$ cells and $\mathrm{B}$ cells) express clonally distributed receptors specific for diverse antigens (allergens) and are the key mediators of immune response. Among $\mathrm{T}$ cells, $\mathrm{CD} 4{ }^{+} \mathrm{T}$ cells are called helper $\mathrm{T}$ cells (Th cells) because they help B cells or phagocytes to produce antibodies or destroy ingested microbes, respectively. Further, $\mathrm{CD} 4^{+} \mathrm{T}$ cells exhibit functionally distinct subsets called Th1, Th2, and Th17 that produce different cytokines and eliminate different types of pathogens.

In allergic disease, epithelial barrier disruption leads to production of innate cytokines such as IL-33 that skew DC phenotypes and activate innate immune cells (e.g., ILC2s, mast cells, and basophils), thereby eventually promoting the 


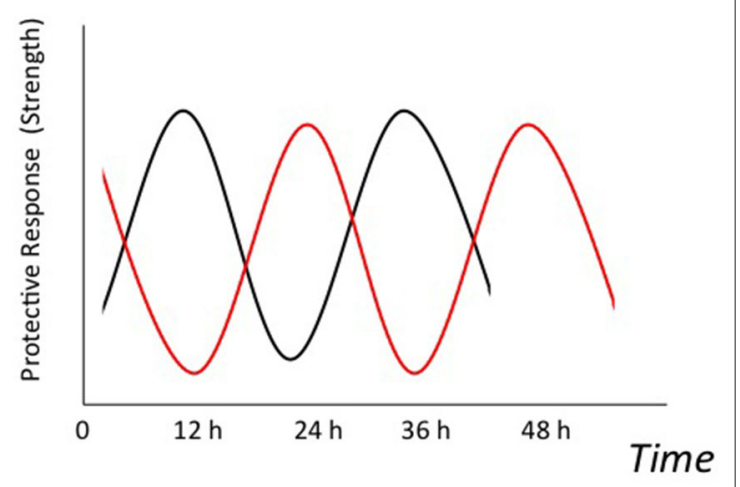

Epithelial Barrier Function

Immune Response Associated with Allergy

FIGURE 2 | Possible temporal relationships between epithelial barrier function and allergic immune response. Epithelial barrier function and immune response associated with allergic disease (allergic immune response) are under control of the circadian clock. We speculate that epithelial barrier function and allergic immune response might temporally complement to each other. Briefly, clock system may maximize allergic immune response at the time-of-day when epithelial barrier function is weakest. This may be because the risk of attack by helminth and biting arthropods is highest at that time.

development of Th2 cell that secrete IL-4, IL-5, and IL-13. These cytokines promote B-cell isotype switching and IgE production, eosinophil accumulation, and mucus production from epithelial cells and shapes allergic inflammation (2).

Clock genes are rhythmically expressed in both $\mathrm{T}$ and $\mathrm{B}$ cells $(82,83)$ and temporally control lymphocyte trafficking and development, and immune responses against diverse pathogens (10-13). However, there have been limited studies addressing the roles of clock in $\mathrm{T}$ and $\mathrm{B}$ cell responses associated with allergic disease.

\section{T Cell-Response}

A mouse model of contact hypersensitivity in the skin depends on Th2 cells. In this model, Clock-mutant mice exhibit severe inflammation and increases in IL-4/IL-13 expression, mast cell number, and serum IgE levels relative to wild-type mice (84). As stated earlier, deletion of Bmal1 in dendritic cells enhances the gut helminth Trichuris muris egg-specific protective Th2 response while suppressing the Th1 response. These findings suggest that clock proteins inhibit Th2 development and function, and that clock disruption may promote the Th2 response.

Th17 cells protect against bacterial and fungal infections at mucosal surfaces. In contrast to Th2 cells, circadian proteins clearly regulate Th17 cell differentiation (85). Deficiency of $\operatorname{ROR} \alpha$ or $\operatorname{ROR} \gamma$ impairs Th17 development in mice. Consistent with this, mice maintained under chronic light cycle perturbations exhibit altered Th17 cell frequencies in the intestines relative to mice maintained under a normal light cycle. Since Th17 cells have been implicated in neutrophilic asthma (86), clock disruption may affect specific phenotypes of asthma.
Regulatory T cells (Tregs) contribute to prevention of allergic disease by regulating effector cells. In humans, genetic deficiency of Tregs shows allergic manifestations (87). Interestingly, mice subjected to perturbed light/dark cycles (6-h advance every 4 days) have fewer Tregs in the intestine than mice subjected to normal light/dark cycles; this is associated with the development of food allergy (88). Consistently, nurses with regular day/nightshift rotation exhibit an increased incidence of food allergy in comparison to nurses with no such rotation of work hours (88). Thus, clock disruption may decrease the number of Tregs, thereby precipitating allergic disease.

\section{B-Cell Response}

The connection between clock and B cell response is not wellstudied. Bmal1-deficient mice have fewer pre-B cells in the bone marrow, as well as fewer B cells in the peripheral blood and spleen (89). This likely involves Bmal1 deficiency in the bone marrow microenvironment, but not B cell-intrinsic Bmal1 $(89,90)$. Thus, Bmall may play a role in the normal differentiation of B cells, but its relevance to allergy remains unknown.

Collectively, these findings suggest that the circadian clock is strongly linked to two fundamental biological aspects of allergic disease, epithelial barrier function and immune responses (Figure 1).

\section{ALLERGIC IMMUNE RESPONSE MAY TEMPORALLY COMPLEMENT EPITHELIAL BARRIER FUNCTION}

Circadian gating of epithelial barrier function and immune response likely evolved to anticipate environmental physical, chemical, and biological insults (e.g., hot air, pollutants, pathogens) and to maximize host defense during the greatest time of the insults' exposure $(11,13)$. The temporal gating can also limit the costs of the defense response and may increase host fitness $(11,13)$.

Allergic immune response, in particular IgE/mast cellmediated response, is thought to evolve to confer protection against macroparasites such as helminth worms and biting arthropods such as mite and mosquito (91). Therefore, it is possible that the circadian control of immune responses associated with allergic disease deal with helminth and biting arthropods that themselves behave in a circadian manner $(92,93)$. We speculate that allergic immune response may temporally complement epithelial barrier function (Figure 2). For instance, skin, intestinal, and possibly airway barrier functions largely weaken in the resting phase $(34,40)$. This may be due to less exposure to noxious stimuli such as hot air and food-borne bacteria and fungi associated with feeding behavior in the resting phase than in the active phase. On the other hand, clock system may maximize mast cell response to IgE during the resting phase $(14,15)$ in order to prepare for the risk of attack by helminth and biting arthropods at that time. In other words, helminth and biting arthropods might evolve to attack target animals at the time-of-day when the animals' epithelial barrier function is weakest. 


\section{CONCLUDING REMARKS: CIRCADIAN-DISRUPTED MODERN LIFESTYLES MAY PROMOTE ALLERGIC DISEASE}

The periodicity of allergic disease impacts on diagnostic and preventive aspects as well as on treatment effects. Recent studies highlight that circadian clock underpins allergic reaction, which likely confers the periodicity of allergic disease (14-16). Importantly, it is becoming clear that the circadian clock is a potent regulator of allergic reaction with more than a simple time-keeping role (16). Of note, several studies suggest that the circadian clock is strongly linked to two fundamental biological aspects of allergic disease, epithelial barrier function and immune responses. Thus, clock disruption can precipitate and enhance disease by deregulating the epithelial barrier and immune functions.

This new knowledge highlights circadian disruption as a new precipitating factor of allergic disease in modern society, in which our sleep, work, and eating habits are out of sync with endogenous circadian rhythmicity. This may partly explain why allergic disease is so prevalent in developed countries. Accordingly, the relationship between circadian biology and

\section{REFERENCES}

1. Reynolds LA, Finlay BB. Early life factors that affect allergy development. Nat Rev Immunol. (2017) 17:518-28. doi: 10.1038/nri.2017.39

2. Hammad H, Lambrecht BN. Barrier epithelial cells and the control of type 2 immunity. Immunity. (2015) 43:29-40. doi: 10.1016/j.immuni.2015.07.007

3. Goleva E, Berdyshev E, Leung DY. Epithelial barrier repair and prevention of allergy. J Clin Invest. (2019) 129:1463-74. doi: 10.1172/JCI124608

4. Palomares O, Akdis M, Martín-Fontecha M, Akdis CA. Mechanisms of immune regulation in allergic diseases: the role of regulatory $\mathrm{T}$ and $\mathrm{B}$ cells. Immunol Rev. (2017) 278:219-36. doi: 10.1111/imr.12555

5. Czarnowicki T, Krueger JG, Guttman-Yassky E. Novel concepts of prevention and treatment of atopic dermatitis through barrier and immune manipulations with implications for the atopic march. J Allergy Clin Immunol. (2017). 139:1723-4. doi: 10.1016/j.jaci.2017.04.004

6. Stelmaszczyk-Emmel A, Zawadzka-Krajewska A, Szypowska A, Kulus M, Demkow U. Frequency and activation of CD4+CD25 FoxP3+ regulatory T cells in peripheral blood from children with atopic allergy. Int Arch Allergy Immunol. (2013) 162:16-24 doi: 10.1159/000350769

7. Turek FW. Circadian clocks: Not your grandfather's clock. Science. (2016) 354:992-3. doi: 10.1126/science.aal2613

8. Matsui MS, Pelle E, Dong K, Pernodet N. Biological rhythms in the skin. Int J Mol Sci. (2016) 17:801. doi: 10.3390/ijms17060801

9. Pagel R, Bär F, Schröder T, Sünderhauf A, Künstner A, Ibrahim $\mathrm{SM}$, et al. Circadian rhythm disruption impairs tissue homeostasis and exacerbates chronic inflammation in the intestine. FASEB J. (2017) 31:470719. doi: 10.1096/fj.201700141RR

10. Cermakian N, Lange T, Golombek D, Sarkar D, Nakao A, Shibata S, Mazzoccoli G. Crosstalk between the circadian clock circuitry and the immune system. Chronobiol Int. (2013) 30:870-88. doi: 10.3109/07420528.2013. 782315

11. Curtis AM, Bellet MM, Sassone-Corsi P, O’Neill LA. Circadian clock proteins and immunity. Immunity. (2014) 40:17886. doi: 10.1016/j.immuni.2014.02.002 allergy will become an important area of research to understand allergic diseases in the modern era and exploring new ways to prevent or treat these disorders. In this context, we propose that lifestyle or therapeutic interventions that align the endogenous circadian clock with environmental cycles should be a part of the efforts to prevent or treat allergic disease in $24 / 7$ society.

\section{AUTHOR CONTRIBUTIONS}

The author confirms being the sole contributor of this work and has approved it for publication.

\section{FUNDING}

This work was supported in part by JSPS KAKEN Grant Number $18 \mathrm{H} 02848$ and 19K22636 from the Ministry of Education, Culture, Sports, Science, and Technology of Japan.

\section{ACKNOWLEDGMENTS}

The author thanks all the members of our laboratory and Ms. Yukino Fukasawa for her valuable general assistance. Regrettably, due to space limitations, I was unable to refer to many important papers in this field.
12. Scheiermann C, Gibbs J, Ince L, Loudon A. Clocking in to immunity. Nat Rev Immunol. (2018) 18:423-37. doi: 10.1038/s41577-018-0008-4

13. Man K, Loudon A, Chawla A. Immunity around the clock. Science. (2016) 354:999-1003. doi: 10.1126/science.aah4966

14. Nakao A, Nakamura Y, Shibata S. The circadian clock functions as a potent regulator of allergic reaction. Allergy. (2015) 70:467-73 doi: 10.1111/all.12596

15. Christ P, Sowa AS, Froy O, Lorentz A. The circadian clock drives mast cell functions in allergic reactions. Front Immunol. (2018) 9:1526. doi: 10.3389/fimmu.2018.01526

16. Nakao A. Clockwork allergy: How the circadian clock underpins allergic reactions. J. Allergy Clin. Immunol. (2018) 142:102131. doi: 10.1016/j.jaci.2018.08.007

17. Plikus MV, Andersen B. Skin as a window to body-clock time. Proc Natl Acad Sci USA. (2018) 115:12095-7. doi: 10.1073/pnas.1817419115

18. Hoogerwerf WA, Hellmich HL, Cornélissen G, Halberg F, Shahinian VB, Bostwick J, et al. Clock gene expression in the murine gastrointestinal tract: endogenous rhythmicity and effects of a feeding regimen. Gastroenterology. (2007) 133:1250-60. doi: 10.1053/j.gastro.2007.07.009

19. Nosal C, Ehlers A, Haspel JA. Why lungs keep time: circadian rhythms and lung immunity. Annu Rev Physiol. (2020) 82:391412. doi: 10.1146/annurev-physiol-021119-034602

20. Takahashi JS. Transcriptional architecture of the mammalian circadian clock. Nat Rev Genet. (2017) 18:164-79. doi: 10.1038/nrg.2016.150

21. Patke A, Young MW, Axelrod S. Molecular mechanisms and physiological importance of circadian rhythms. Nat Rev Mol Cell Biol. (2020) 21:6784. doi: 10.1038/s41580-019-0179-2

22. Zhao $\mathrm{X}$, Cho $\mathrm{H}$, Yu RT, Atkins AR, Downes $M$, Evans RM. Nuclear receptors rock around the clock. EMBO Rep. (2014) 15:518-28. doi: 10.1002/embr.201338271

23. Zhang R, Lahens NF, Ballance HI, Hughes ME, Hogenesch JB. A circadian gene expression atlas in mammals: implications for biology and medicine. Proc Natl Acad Sci USA. (2014). 111:16219-24. doi: 10.1073/pnas.1408886111

24. Schibler U, Gotic I, Saini C, Gos P, Curie T, Emmenegger Y, et al Clock-talk: interactions between central and peripheral circadian oscillators 
in mammals. Cold Spring Harb Symp Quant Biol. (2015) 80:22332. doi: $10.1101 / \mathrm{sqb} .2015 .80 .027490$

25. Panda S. Circadian physiology of metabolism. Science. (2016) 354:100815. doi: 10.1126/science.aah4967

26. Stokkan KA, Yamazaki S, Tei H, Sakaki Y, Menaker M. Entrainment of the circadian clock in the liver by feeding. Science. (2001) 291:4903. doi: $10.1126 /$ science. 291.5503 .490

27. Roenneberg T, Merrow M. The circadian clock and human health. Curr Biol. (2016) 26:R432-43. doi: 10.1016/j.cub.2016.04.011

28. Walker WH 2nd, Walton JC, DeVries AC, Nelson RJ. Circadian rhythm disruption and mental health. Transl Psychiatry. (2020) 10:28. doi: 10.1038/s41398-020-0694-0

29. Sulli G, Manoogian ENC, Taub PR, Panda S. Training the circadian clock, clocking the drugs, and drugging the clock to prevent, manage, and treat chronic diseases. Trends Pharmacol Sci. (2018) 39:812-27 doi: 10.1016/j.tips.2018.07.003

30. Schleimer RP, Berdnikovs S. Etiology of epithelial barrier dysfunction in patients with type 2 inflammatory diseases. J Allergy Clin Immunol. (2017) 139:1752-1761. doi: 10.1016/j.jaci.2017.04.010

31. Tognini P, Murakami M, Sassone-Corsi P. Interplay between Microbes and the Circadian Clock. Cold Spring Harb Perspect Biol. (2018) 10:a028365. doi: $10.1101 /$ cshperspect.a028365

32. Schmalle V, Lorentz A. Role of the microbiota in circadian rhythms of the host. Chronobiol Int. (2020) 37:310-310. doi: 10.1080/07420528.2020.1726374

33. Murakami M, Tognini P. The circadian clock as an essential molecular link between host physiology and microorganisms. Front Cell Infect Microbiol. (2020) 9:469. doi: 10.3389/fcimb.2019.00469

34. Vaughn AR, Clark AK, Sivamani RK, Shi VY. Circadian rhythm in atopic dermatitis-Pathophysiology and implications for chronotherapy. Pediatr Dermatol. (2018) 35:152-7. doi: 10.1111/pde.13364

35. Nakajima S, Nomura T, Common J, Kabashima K. Insights into atopic dermatitis gained from genetically defined mouse models. J Allergy Clin Immunol. (2019) 143:13-25. doi: 10.1016/j.jaci.2018.11.014

36. Matsunaga $\mathrm{N}$, Itcho $\mathrm{K}$, Hamamura $\mathrm{K}$, Ikeda E, Ikeyama H, Furuichi $\mathrm{Y}$, Watanabe $\mathrm{M}$, et al. 24-hour rhythm of aquaporin-3 function in the epidermis is regulated by molecular clocks. J Invest Dermatol. (2014) 134:163644. doi: $10.1038 /$ jid.2014.13

37. Peterson LW, Artis D. Intestinal epithelial cells: regulators of barrier function and immune homeostasis. Nat Rev Immunol. (2014) 14:14153. doi: $10.1038 /$ nri3608

38. Perrier C, Corthésy B. Gut permeability and food allergies. Clin Exp Allergy. (2011) 41:20-8. doi: 10.1111/j.1365-2222.2010.03639.x

39. Buckley A, Turner JR. Cell biology of tight junction barrier regulation and mucosal disease. Cold Spring Harb Perspect Biol. (2018) 10:a029314. doi: 10.1101/cshperspect.a029314

40. Kyoko OO, Kono H, Ishimaru K, Miyake K, Kubota T, Ogawa H, et al. Expressions of tight junction proteins occludin and claudin-1 are under the circadian control in the mouse large intestine: implications in intestinal permeability and susceptibility to colitis. PLoS ONE. (2014) 9:e98016. doi: 10.1371/journal.pone.0098016

41. Tanabe K, Kitagawa E, Wada M, Haraguchi A, Orihara K, Tahara Y, et al. Antigen exposure in the late light period induces severe symptoms of food allergy in an OVA-allergic mouse model. Sci Rep. (2015) 5:14424. doi: 10.1038/srep14424

42. Summa KC, Voigt RM, Forsyth CB, Shaikh M, Cavanaugh K, et al. Disruption of the circadian clock in mice increases intestinal permeability and promotes alcohol-induced hepatic pathology and inflammation. PLOS ONE. (2013) 8:e67102 doi: 10.1371/journal.pone.0067102

43. Xiao C, Puddicombe SM, Field S, Haywood J, Broughton-Head V, Puxeddu I, et al. Defective epithelial barrier function in asthma. J Allergy Clin Immunol. (2011) 128:549-56. doi: 10.1016/j.jaci.2011.05.038

44. Holgate ST. Epithelium dysfunction in asthma. $J$ Allergy Clin Immunol. (2007) 120:1233-44. doi: 10.1016/j.jaci.2007. 10.025

45. Gibbs J, Ince L, Matthews L, Mei J, Bell $\mathrm{T}$, Yang $\mathrm{N}$, et al. An epithelial circadian clock controls pulmonary inflammation and glucocorticoid action. Nat Med. (2014) 20:919-26. doi: 10.1038/nm. 3599
46. Braun-Fahrlander C, Riedler J, Herz U, Eder W, Waser M, Grize L, et al. Environmental exposure to endotoxin and its relation to asthma in school-age children. N Engl J Med. (2002) 347:869-77. doi: 10.1056/NEJMoa020057

47. Hammad H, Chieppa M, Perros F, Willart MA, Germain RN, Lambrecht BN. House dust mite allergen induces asthma via Toll-like receptor 4 triggering of airway structural cells. Nat Med. (2009) 15:410-6. doi: 10.1038/nm.1946

48. Pariollaud M, Gibbs JE, Hopwood TW, Brown S, Begley N, Vonslow $\mathrm{R}$, et al. Circadian clock component REV-ERB $\alpha$ controls homeostatic regulation of pulmonary inflammation. J Clin Invest. (2018) 128:228196. doi: 10.1172/JCI93910

49. Ehlers A, Xie W, Agapov E, Brown S, Steinberg D, Tidwell R, et al. BMAL1 links the circadian clock to viral airway pathology and asthma phenotypes. Mucosal Immunol. (2018) 11:97-111. doi: 10.1038/mi.2017.24

50. Galli, SJ, Tsai M. IgE and mast cells in allergic diseases. Nat Med. (2012) 18:693-704. doi: 10.1038/nm.2755

51. Smolensky MH, Lemmer B, Reinberg AE. Chronobiology and chronotherapy of allergic rhinitis and bronchial asthma. Adv Drug Deliv Rev. (2007) 59:85282. doi: 10.1016/j.addr.2007.08.016

52. Reinberg A, Zagula-Mally Z, Ghata J, Halberg F. Circadian reactivity rhythm of human skin to house dust, penicillin, and histamine. J Allergy. (1969) 44:292-306 doi: 10.1016/0021-8707(69)90034-3

53. Nakamura $Y$, Nakano N, Ishimaru K, Hara M, Ikegami T, Tahara $Y$, et al. Circadian regulation of allergic reactions by the mast cell clock in mice. $J$ Allergy Clin Immunol. (2014) 133:568-75. doi: 10.1016/j.jaci.2013.07.040

54. Ando N, Nakamura Y, Ishimaru K, Ogawa H, Okumura K, Shimada S, et al. Allergen-specific basophil reactivity exhibits daily variations in seasonal allergic rhinitis. Allergy. (2015) 70:319-22. doi: 10.1111/all.12552

55. Spadaro G, Giurato G, Stellato C, Marone G, Casolaro V. Basophil degranulation in response to IgE ligation is controlled by a distinctive circadian clock in asthma. Allergy. (2020) 75:158-68. doi: 10.1111/all.14002

56. Lind C, Skaarup SH, Lorentz A, Hoffmann HJ. Basophil testing with CD63 in pollen-sensitized patients is independent of the circadian clock. J Allergy Clin Immunol. (2018) 141:1906-8. doi: 10.1016/j.jaci.2017.10.048

57. Nakamura Y, Harama D, Shimokawa N, Hara M, Suzuki R, Tahara Y, et al. Circadian clock gene period2 regulates a time-of-day-dependent variation in cutaneous anaphylactic reaction. J Allergy Clin Immunol. (2011). 127:103845.e1-3. doi: 10.1016/j.jaci.2011.02.006

58. Nakamura Y, Nakano N, Ishimaru K, Ando N, Katoh R, Suzuki-Inoue K, et al. Inhibition of IgE-mediated allergic reactions by pharmacologically targeting the circadian clock. J Allergy Clin Immunol. (2016) 137:122635. doi: 10.1016/j.jaci.2015.08.052

59. Nakamura Y, Ishimaru K, Nakao A. Time-restricted feeding in rest phase alters IgE/mast cell-mediated allergic reaction in mice. Allergol Int. (2019) 69:296-99. doi: 10.1016/j.alit.2019.09.004

60. Ohno T, Morita H, Arae K, Matsumoto K, Nakae S. Interleukin-33 in allergy. Allergy. (2012) 67:1203-14. doi: 10.1111/all.12004

61. Kawauchi, T, Ishimaru K, Nakamura Y, Nakano N, Hara M, et al. Clockdependent temporal regulation of IL-33/ST2-mediated mast cell response. Allergol Int. (2017) 66:472-8. doi: 10.1016/j.alit.2017.02.004

62. Chusid MJ. Eosinophils: Friends or foes? J Allergy Clin Immunol Pract. (2018) 6:1439-44. doi: 10.1016/j.jaip.2018.04.031

63. Pelaia C, Paoletti G, Puggioni F, Racca F, Pelaia G, Canonica GW, et al. Interleukin-5 in the pathophysiology of severe asthma. Front Physiol. (2019) 10:1514.

64. Baumann A, Gönnenwein S, Bischoff SC, Sherman H, Chapnik N, Froy $\mathrm{O}$, et al. The circadian clock is functional in eosinophils and mast cells. Immunology. (2013) 140:465-74. doi: 10.1111/imm.12157

65. Baumann A, Feilhauer K, Bischoff SC, Froy O, Lorentz A. IgE-dependent activation of human mast cells and fMLP-mediated activation of human eosinophils is controlled by the circadian clock. Mol Immunol. (2015) 64:7681. doi: 10.1016/j.molimm.2014.10.026

66. Dahl R, Venge P, Olsson I. Blood eosinophil leucocytes and eosinophil cationic protein. diurnal variation in normal subjects and patients with bronchial asthma. Scand J Respir Dis. (1978) 59:323-5. doi: 10.1175/1520-0477-59.3.323

67. Durrington HJ, Gioan-Tavernier GO, Maidstone RJ, Krakowiak K, Loudon ASI, Blaikley JF, et al. Time of day affects eosinophil biomarkers in asthma: implications for diagnosis and treatment. Am J Respir Crit Care Med. (2018) 198:1578-81. doi: 10.1164/rccm.201807-1289LE 
68. Nussbaum JC, van Dyken SJ, von Moltke J, Cheng LE, Mohapatra A, Molofsky $\mathrm{AB}$, et al. Type 2 innate lymphoid cells control eosinophil homeostasis. Nature. (2013) 502:245-8. doi: 10.1038/nature12526

69. Shapouri-Moghaddam A, Mohammadian S, Vazini H, Taghadosi M, Esmaeili SA, Mardani F, et al. Macrophage plasticity, polarization, and function in health and disease. J Cell Physiol. (2018) 233:6425-40. doi: 10.1002/jcp.26429

70. Hayashi M, Shimba S, Tezuka M. Characterization of the molecular clock in mouse peritoneal macrophages. Biol Pharm Bull. (2007) 30:6216. doi: $10.1248 / \mathrm{bpb} .30 .621$

71. Keller M, Mazuch J, Abraham U, Eom GD, Herzog ED, Volk HD, et al. A circadian clock in macrophages controls inflammatory immune responses. Proc Natl Acad Sci USA. (2009) 106:21407-12. doi: 10.1073/pnas.0906361106

72. Kitchen GB, Cunningham PS, Poolman TM, Iqbal M, Maidstone R, Baxter $\mathrm{M}$, et al. The clock gene Bmall inhibits macrophage motility, phagocytosis, and impairs defense against pneumonia. Proc Natl Acad Sci USA. (2020) 117:1543-51. doi: 10.1073/pnas.1915932117

73. Zasłona Z, Case S, Early JO, Lalor SJ, McLoughlin RM, Curtis AM, et al. The circadian protein BMAL1 in myeloid cells is a negative regulator of allergic asthma. Am J Physiol Lung Cell Mol Physiol. (2017) 312:L85560. doi: 10.1152/ajplung.00072.2017

74. Morita H, Moro K, Koyasu S. Innate lymphoid cells in allergic and nonallergic inflammation. J Allergy Clin Immunol. (2016) 138:125364. doi: 10.1016/j.jaci.2016.09.011

75. Bartemes KR, Kephart GM, Fox SJ, Kita H. Enhanced innate type 2 immune response in peripheral blood from patients with asthma. J Allergy Clin Immunol. (2014) 134:671-8.e4. doi: 10.1016/j.jaci.2014.06.024

76. Halim TY, MacLaren A, Romanish MT, Gold MJ, McNagny KM, Takei F. Retinoic-acid-receptor-related orphan nuclear receptor alpha is required for natural helper cell development and allergic inflammation. Immunity. (2012) 37:463-74. doi: 10.1016/j.immuni.2012.06.012

77. Wang Q, Robinette ML, Billon C, Collins PL, Bando JK, Fachi JL, et al. Circadian rhythm-dependent and circadian rhythm-independent impacts of the molecular clock on type 3 innate lymphoid cells. Sci Immunol. (2019) 4:eaay7501. doi: 10.1126/sciimmunol.aay7501

78. Teng F, Goc J, Zhou L, Chu C, Shah MA, Eberl G, et al. A circadian clock is essential for homeostasis of group 3 innate lymphoid cells in the gut. Sci Immunol. (2019) 4:eaax1215. doi: 10.1126/sciimmunol.aax1215

79. Everaere L, Ait-Yahia S, Molendi-Coste O, Vorng H, Quemener S, $\mathrm{LeVu} \mathrm{P}$, et al. Innate lymphoid cells contribute to allergic airway disease exacerbation by obesity. J Allergy Clin Immunol. (2016) 138:130918.e11. doi: 10.1016/j.jaci.2016.03.019

80. Lu N, Li Y, Zhang Z, Xing J, Sun Y, Yao S, et al. Human semaphorin-4A drives Th2 responses by binding to receptor ILT-4. Nat Commun. (2018) 9:742. doi: 10.1038/s41467-018-03128-9

81. Hopwood TW, Hall S, Begley N, Forman R, Brown S, Vonslow $\mathrm{R}$, et al. The circadian regulator BMAL1 programmes responses to parasitic worm infection via a dendritic cell clock. Sci Rep. (2018) 8:3782. doi: 10.1038/s41598-018-22021-5
82. Bollinger T, Leutz A, Leliavski A, Skrum L, Kovac J, Bonacina L, et al. Circadian clocks in mouse and human CD4+ T cells. PLoS ONE. (2011) 6:e29801. doi: 10.1371/journal.pone.0029801

83. Silver AC, Arjona A, Hughes ME, Nitabach MN, Fikrig E. Circadian expression of clock genes in mouse macrophages, dendritic cells, and B cells. Brain Behav Immun. (2012) 26:407-13. doi: 10.1016/j.bbi.2011. 10.001

84. Takita E, Yokota S, Tahara Y, Hirao A, Aoki N, Nakamura Y, et al. Biological clock dysfunction exacerbates contact hypersensitivity in mice. $\mathrm{Br}$ J Dermatol. (2013). 168:39-46. doi: 10.1111/j.1365-2133.2012.11176.x

85. Yu X, Rollins D, Ruhn KA, Stubblefield JJ, Green CB, Kashiwada M, et al. TH17 cell differentiation is regulated by the circadian clock. Science. (2013) 342:727-30. doi: 10.1126/science. 1243884

86. Boonpiyathad T, Sözener ZC, Satitsuksanoa P, Akdis CA Immunologic mechanisms in asthma. Semin Immunol. (2019) 46:101333. doi: 10.1016/j.smim.2019.101333

87. Torgerson TR, Linane A, Moes N, Anover S, Mateo V, Rieux-Laucat F, et al. Severe food allergy as a variant of IPEX syndrome caused by a deletion in a noncoding region of the FOXP3 gene. Gastroenterology. (2007) 132:170517. doi: 10.1053/j.gastro.2007.02.044

88. Yang G, Zhang H, Liu Y, Feng Y, Luo XQ, Liu ZQ, et al. Alternation of circadian clock modulates forkhead box protein-3 gene transcription in CD4+ T cells in the intestine. J Allergy Clin Immunol. (2016) 138:14469.e10. doi: 10.1016/j.jaci.2016.04.035

89. Sun Y, Yang Z, Niu Z, Peng J, Li Q, Xiong W, et al. MOP3, a component of the molecular clock, regulates the development of B cells. Immunology. (2006) 119:451-60. doi: 10.1111/j.1365-2567.2006.02456.x

90. Hemmers S, Rudensky AY. The cell-intrinsic circadian clock is dispensable for lymphocyte differentiation and function. Cell Rep. (2015) 11:133949. doi: 10.1016/j.celrep.2015.04.058

91. Pulendran B, Artis D. New paradigms in type 2 immunity. Science. (2012) 337:431-5. doi: 10.1126/science. 1221064

92. Hawkins F. Circadian and other rhythms of parasites. Adv Parasitol. (1975) 13:123-82. doi: 10.1016/S0065-308X(08)60320-6

93. Carvalho Cabral P, Olivier M, Cermakian N. The complex interplay of parasites, their hosts, and circadian clocks. Front Cell Infect Microbiol. (2019) 9:425. doi: 10.3389/fcimb.2019.00425

Conflict of Interest: The author declares that the research was conducted in the absence of any commercial or financial relationships that could be construed as a potential conflict of interest.

Copyright (c) 2020 Nakao. This is an open-access article distributed under the terms of the Creative Commons Attribution License (CC BY). The use, distribution or reproduction in other forums is permitted, provided the original author(s) and the copyright owner(s) are credited and that the original publication in this journal is cited, in accordance with accepted academic practice. No use, distribution or reproduction is permitted which does not comply with these terms. 